\title{
Investigação Experimental do Desempenho de um Regulador Automático de Tensão Digital Aplicado a um Sistema de Microgeração de Energia Elétrica
}

\author{
Diogo Augusto Nascimento e Araújo*. Renan Landau Paiva de Medeiros.* \\ João Edgar Chaves FIlho*. Florindo Antônio de Carvalho Ayres Júnior*. Iury Valente de Bessa*. \\ * Departamento de Eletricidade, Faculdade de Tecnologia, Universidade Federal do Amazonas, Manaus-AM, Brasil \\ (e-mails:daraujo.augusto@gmail.com,renanlandau@ufam.edu.br,joaoedgarc@gmail.com, \\ florindoayres@ufam.edu.br,iury.bessa@gmail.com).
}

\begin{abstract}
This work presents the development of an Automatic Voltage Regulator (AVR) applied to a low-voltage power energy system, for the realization of this, digital control concepts and techniques were used. The control and regulation system was developed and embedded in the Arduino Due development platform, a microcontroller considered of high performance. The signal sent by the controller is used in a DC-DC power converter Buck topology to control its average voltage value which is used to power the field coil of the synchronous generator, thus providing the electrical excitation required for the creation of a magnetic field. This control is done by varying the working cycle of the static switch, which allows a variation of the average voltage of the DC-DC power converter element of the Buck type, this information is the result of the calculation in the microcontroller, and with this it is used the pulse width modulation (PWM) technique to perform the static key switch.. The results show that the controlled system was successful in presenting the desired performance by means of simulation and experimental tests.
\end{abstract}

Resumo: Neste trabalho é apresentado o desenvolvimento de um Regulador Automático de Tensão (RAT) aplicado a um sistema de microgeração de energia. O sistema de controle e regulação foi desenvolvido e embarcado de controle e regulação foi desenvolvida e embarcada na plataforma de desenvolvimento do Arduino Due, um microcontrolador considerado de alto desempenho. O sinal enviado pelo controlador é utilizado em um conversor de potência CC-CC de topologia Buck, para controlar o seu valor médio de tensão que é utilizado para alimentar a bobina de campo do gerador síncrono, fornecendo, assim, a excitação elétrica necessária para a criação de um campo magnético. Esse controle é feito através da variação do ciclo de trabalho da chave estática, o que permite uma variação da tensão média do elemento conversor de potência CC-CC do tipo Buck, tal informação é resultado do cálculo no microcontrolador, e com isso utiliza-se a técnica de modulação por largura de pulsos (PWM), para realizar o chaveamento da chave estática. Os resultados mostram que o sistema controlado, obteve êxito com relação a apresentar o desempenho desejado por meio da realização de testes de simulação e experimentais.

Keywords: Digital Control; Automatic Voltage Regulation; Synchronous Generator; DC-DC Power Converter; Buck;

Palavras-chaves: Controle Digital; Regulação Automática de Tensão; Gerador Síncrono; Conversor de Potência CC-CC; Buck;

\section{INTRODUÇÃO}

Devido ao aumento da demanda de energia elétrica para os mais diversos tipos de aplicações, os sistemas elétricos de potência têm se tornado cada vez mais requisitados e cada vez mais complexos. Para a análise desse tipo de sistema, observam-se fatores que podem ser melhorados ou corrigidos sempre com o objetivo de fornecer energia elétrica com os menores custos possíveis, com qualidade e com menor desperdício de energia (COSTA et al., 2012). Um requisito importante na geração de energia elétrica é a confiabilidade do sistema, deste modo, torna-se necessário constituir estratégias que garantam o funcionamento do sistema elétrico dentro das especificações, para o caso da geração de energia elétrica, neste caso em específico, os sistemas de controle para a regulação da tensão terminal da máquina síncrona tornam-se imprescindíveis (KUNDUR, 1994)(NASCIMENTO FILHO, 2011)(FERNANDES, 2011).

A realização de testes e estudos em sistemas de potência reais envolve restrições e riscos, tanto para o sistema de energia quanto para as pessoas. Diante das dificuldades em se realizar ensaios em sistemas de grande porte para se avaliar o desempenho dos controladores projetados, torna-se importante o desenvolvimento deste estudo, cuja principal contribuição é a investigação experimental do desempenho de um Regulador Automático de Tensão digital em um sistema real. Este tipo de teste é de grande importância científica, pois permite identificar possíveis fragilidades no sistema de controle projetado, as quais normalmente não podem ser observadas ou reproduzidas via simulação computacional. Os testes no sistema de potência real, em laboratório, permitem observar os efeitos prejudiciais de dinâmicas não modeladas, tais como: não-linearidades de sensores, saturação, histerese, 
contaminação por harmônicos da componente fundamental, ruídos produzidos pelo chaveamento dos atuadores.

(MORAES, 2011)

Diversos estudos recentes como (EKINCI et al, 2019) e (CALDERON, 2017) tem como cerne de suas pesquisas, a investigação de estratégias de controle para projeto de um Regulador Automático de Tensão (RAT) com o intuito de obter melhorias de desempenho dinâmico em sistemas de microgeração e que desenvolvem diversos métodos de projeto destes controladores, objetivando melhor solucionar os problemas da regulação de tensão em unidades de microgeração, bem como uma investigação computacional de seus desempenhos. Os Sistemas Elétricos de Potência em escala de laboratório são valiosas ferramentas para o desenvolvimento de pesquisas e estudos de técnicas de regulação aplicadas no controle das unidades geradoras, e de fenômenos presentes nos grandes sistemas de potência (MORAES, 2011).

O regulador automático de tensão (RAT) é um equipamento essencial usado em sistemas de potência. Como o próprio nome indica, o objetivo principal do RAT é controlar a tensão do terminal do gerador síncrono, ajustando a tensão do excitador. Devido à alta indutância dos enrolamentos de campo do gerador e variações de carga, é difícil obter uma resposta estável e rápida do regulador. Portanto, é importante melhorar o desempenho do RAT e garantir uma resposta estável e eficiente às mudanças transitórias na tensão do terminal (EKINCI et al., 2019).

O restante deste trabalho está organizado como segue: na Seção 2, é apresentado um referencial teórico tendo uma breve descrição sobre a problemática de regulação automática de tensão, na Seção 3, é realizada uma breve revisão sobre os conceitos de teoria de controle e técnicas de discretização; na Seção 4 é descrito com maiores detalhes a metodologia abordada neste estudo, apresentando o projeto de controle desenvolvido.

\section{VISÃO GERAL}

\subsection{A máquina síncrona}

A máquina síncrona é a principal máquina utilizada para realizar a conversão de outras formas de energia em energia elétrica, devido a aspectos técnicos referentes a seu funcionamento, como sua operação na velocidade síncrona e sua facilidade quanto ao uso de excitatrizes estáticas. O funcionamento do gerador síncrono é fundamentado principalmente pela lei de indução de Faraday. Um campo magnético variante no tempo, produzido pelo movimento angular do rotor, induz uma tensão alternada no estator. $\mathrm{O}$ rotor é alimentado com corrente contínua, assim, a frequência das correntes geradas no estator é proporcional a velocidade angular do rotor (UMANS, 2015).

O enrolamento de campo é alimentado por um elemento de corrente contínua, chamada excitatriz. A função básica do sistema de excitação é fornecer corrente contínua para a bobina de excitação do gerador síncrono. Além disso, o mesmo pode exercer funções de controle para um desempenho satisfatório do sistema de potência ao controlar a tensão de excitação da bobina de campo da máquina síncrona (KUNDUR, 1994)(SAUER, 1998).
A rigor, o modelo de gerador a ser desenvolvido a seguir aplica-se apenas para a condição de máquina a vazio, ou seja, as correntes de fase, $i_{a}, i_{b}$ e $i_{c}$ são iguais a zero, o modelo a ser desenvolvido para a máquina a vazio é uma boa aproximação para representar de forma adequada a dinâmica investigada, mesmo quando o sistema estiver operando sob carga. A indutância do rotor varia de acordo com a posição do rotor, por este motivo, se representa o modelo da máquina síncrona em coordenadas dq0, representado na Figura 1. (KUNDUR, 1994)(SAUER, 1998)

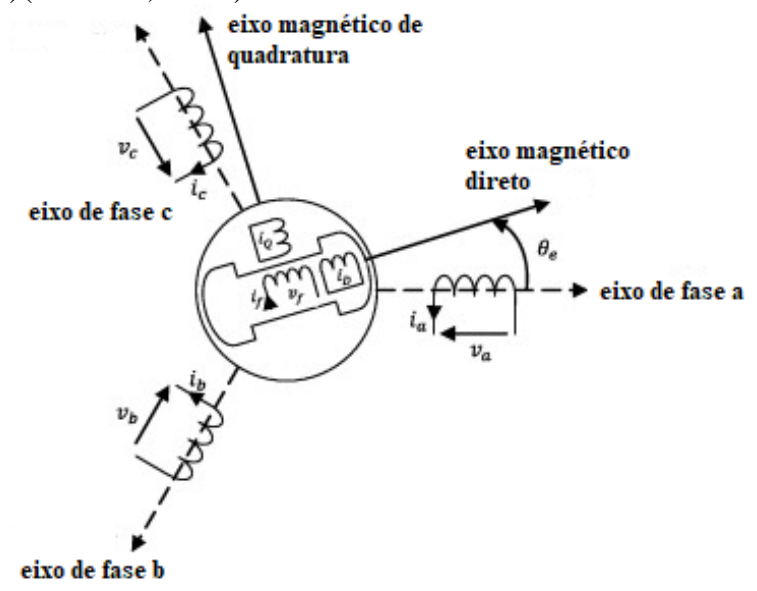

Figura 1 Representação das coordenadas de eixo $a b c$ e $d q 0$ (Adaptado de (UMANS, 2014))

Dessa forma, também se anulam as correntes de eixo-direto $\left(i_{d}=0\right)$ e de eixo-quadratura $\left(i_{q}=0\right)$, já que estas estão relacionadas às correntes de fase, o que também elimina os efeitos das indutâncias síncronas de eixo-direto $\left(L_{d}\right)$ e de eixo de quadratura $\left(L_{q}\right)$, sobre os respectivos fluxos magnéticos $\left(\psi_{d}\right.$ e $\left.\psi_{q}\right)$. Algo que também vem a ser desprezado, são as parcelas de fluxo relacionadas ao efeito das correntes nos enrolamentos amortecedores $\left(i_{k d} e i_{k q}\right)$ sobre suas indutâncias ( $L_{a k d}$ e $L_{a k q}$ ) (MORAES, 2011).

Deste modo, o equacionamento contendo a função de transferência do gerador é obtido conforme apresenta (1):

$$
\frac{v_{t}(s)}{v_{f d}(s)}=\frac{K_{G}}{1+s T_{G}}
$$

Observa-se, na condição de máquina a vazio, a constante de tempo $T_{G}$, representa a constante de tempo transitória de eixo direto em circuito aberto do gerador, denotada por $T_{d o}^{\prime}$ na teoria de máquinas síncronas. Se o modelo acima for aplicado como uma aproximação para o caso de máquina em carga, contudo, $T_{G}$ terá um valor menor que a constante $T_{d o}^{\prime}$ do gerador, e $K_{G}$ é valor de ganho que representa a relação entre a tensões em regime permanente (MORAES, 2011) (KUNDUR, 1994).

\subsection{Problema de Regulação de Tensão}

O controle da tensão é fundamental para manter a confiabilidade e a qualidade da energia elétrica, proporcionando o bom funcionamento dos sistemas elétricos de potência. A tensão terminal do gerador síncrono é controlada pelo RAT, o qual utiliza a medida local de tensão de terminal do gerador. Os valores medidos são comparados 
com um valor de referência de tensão. O sinal de erro passa por um controlador e este é aplicado sobre a excitatriz, que tem o papel de atuador no sistema de geração de energia elétrica A excitatriz controla a tensão sobre a bobina de campo, a qual tem impacto direto sobre a corrente de eixo $d$ e $q$ gerada, conforme apresentado na Figura 1.

Do ponto de vista do sistema de potência, a principal função de um regulador automático de tensão (RAT) é controlar a tensão terminal do gerador síncrono por meio do ajuste de sua excitação de campo.

Um sistema de excitação rápida é monumental para melhorar o desempenho geral do sistema, especialmente durante condições transitórias, bem como para garantir a estabilidade durante falhas e perturbações do sistema. Além disso, durante a partida do motor de alta potência, as quedas de tensão do gerador e as perdas de aquecimento podem ser reduzidas em grande parte por um sistema de excitação rápida (GHAMRI, 2019.

No entanto, as características, tempos de resposta quase instantâneos e altos ganhos do regulador automático de tensão (RAT), que lhe permitem contribuir positivamente para a estabilidade transitória, podem contribuir negativamente para o amortecimento natural do sistema, podendo sendo uma das principais causas de oscilações mal amortecidas em sistemas elétricos de potência, geralmente essa redução no amortecimento do sistema é compensada de forma eficaz por meio da incorporação de estabilizadores do sistema de potência (PSS) (CALDERON, 2017).

\subsection{Excitatriz estática de topologia conversor Buck}

O circuito atuador é um conversor CC-CC do tipo abaixador (conversor do tipo Buck). O objetivo deste circuito é implementar o sistema de excitação do gerador síncrono. A etapa de chaveamento é formada por um IGBT, controlado por um circuito de comando que recebe um sinal PWM (do inglês, Pulse Width Modulation) enviado pelo RAT. O IGBT apresenta em seu encapsulamento um diodo de proteção. $\mathrm{Na}$ Figura 2, é ilustrado o diagrama esquemático do circuito atuador.

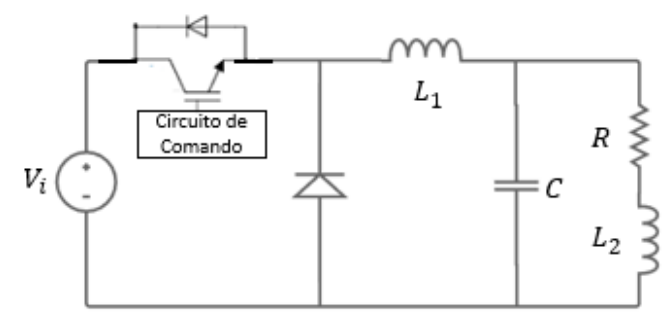

Figura 2 Esquema elétrico do circuito do atuador

\subsubsection{Espaço de estados médio}

A modelagem em tempo contínuo do espaço de estados desse tipo de conversor, ao longo de um período de comutação $T$ com um ciclo de trabalho $d$; requer dois conjuntos de equações diferenciais: um conjunto descreve a operação do circuito durante o intervalo de tempo $0 \leq t \leq d T$, quando os interruptores estão ligados e o outro descreve para quando os interruptores estão desligados, durante o intervalo de tempo $(1-d) T<t \leq T$ (BARBI, 2015).

A matriz de estados do sistema, será a soma ponderada dos estados de cada um dos dois modos de operação. Nesse caso, se o circuito no estado 'ligado' possui as matrizes de estado $A_{1}$ e $B_{1}$ (2); e o estado 'desligado' possui as matrizes de estado $A_{2}$ e $B_{2}(3)$,

$$
\begin{gathered}
{\left[\begin{array}{c}
\dot{V}_{c} \\
\dot{i}_{L 1} \\
\dot{i_{L 2}}
\end{array}\right]=\left[\begin{array}{ccc}
0 & 1 / C & -1 / C \\
-1 / L_{1} & 0 & 0 \\
1 / L_{2} & 0 & -R / L_{2}
\end{array}\right]\left[\begin{array}{c}
V_{c} \\
i_{L 1} \\
i_{L 2}
\end{array}\right]+\left[\begin{array}{c}
0 \\
1 / L_{1} \\
0
\end{array}\right] V_{i n}} \\
y=\left[\begin{array}{lll}
1 & 0 & 0
\end{array}\right]+[0] V_{\text {in }} \\
{\left[\begin{array}{c}
\dot{V}_{C} \\
\dot{i_{L 1}} \\
\dot{i_{L 2}}
\end{array}\right]=\left[\begin{array}{ccc}
0 & 1 / C & -1 / C \\
-1 / L_{1} & 0 & 0 \\
1 / L_{2} & 0 & -R / L_{2}
\end{array}\right]\left[\begin{array}{l}
V_{C} \\
i_{L 1} \\
i_{L 2}
\end{array}\right]+\left[\begin{array}{l}
0 \\
0 \\
0
\end{array}\right] V_{\text {in }}} \\
y=\left[\begin{array}{lll}
1 & 0 & 0
\end{array}\right]+[0] V_{i n}
\end{gathered}
$$

Então a representação final que compreende a dinâmica durante todo o tempo de operação $T$ pode ser apresentada conforme é mostrada em (4).

$$
\dot{x}=\left[\bar{A}_{1} d+\bar{A}_{2}(1-d)\right] \bar{x}+\left[\bar{B}_{1} d+\bar{B}_{2}(1-d)\right] \bar{u}
$$

\subsubsection{Função de transferência}

De posse do modelo matemático em espaço de estados médio, que descreve adequadamente o comportamento do conversor CC-CC do tipo Buck, ao longo de todo o período de chaveamento, pode-se obter a função de transferência, que descreve este modelo, assim como apresenta (5).

$$
\frac{V_{o}}{d}=\frac{V_{\text {in }}\left(\frac{1}{C L_{1}} s+\frac{R}{C L_{1} L_{2}}\right)}{s^{3}+\frac{R}{L_{2}} s^{2}+\left(\frac{1}{C L_{1}}+\frac{1}{C L_{2}}\right) s+\frac{R}{C L_{1} L_{2}}}
$$

\section{METODOLOGIA}

\subsection{Obtenção dos parâmetros do sistema}

O modelo que descreve o comportamento de um gerador síncrono, no que se refere ao comportamento da tensão de armadura em função da tensão aplicada no campo é dado por (1). Porém, a equação encontrada depende ainda de parâmetros construtivos da máquina. Assim, faz-se necessário o uso de algumas estratégias de identificação de parâmetros.

O coeficiente de ganho $\left(K_{g}\right)$ e a constante de tempo $\left(T_{g}\right)$ foram obtidas de forma experimental, com o gerador em velocidade nominal e em vazio. Para a obtenção de $K_{g}$, aplicou-se um sinal de tensão $\mathrm{CC}, E_{f d}$, no enrolamento de campo e realizou-se uma medição, por meio de um multímetro, a tensão terminal eficaz, $V_{t}$, na saída do gerador em regime permanente. $O$ valor deste sinal de tensão $E_{f d}$ para $V_{t}=220 \mathrm{~V}$ 
é de 16,2V. Logo, considerando o regime permanente e aplicando esses valores em (1), obtém-se o valor de $K_{g}=$ 13,58. Em seguida, com o gerador em condições nominais de tensão e velocidade, somou-se um degrau positivo de $10 \%$ de $E_{f d}$ e registrou-se uma curva de resposta ao degrau, nela podese observar que a curva de tensão terminal, desconsiderando o atraso, atingiu $63,2 \%$ do valor de regime permanente em $t=$ $0,55 \mathrm{~s}$, uma representação desse teste é mostrado na Figura 3. Dessa forma, tem-se que a constante de tempo $T_{g}=0,55 \mathrm{~s}$.

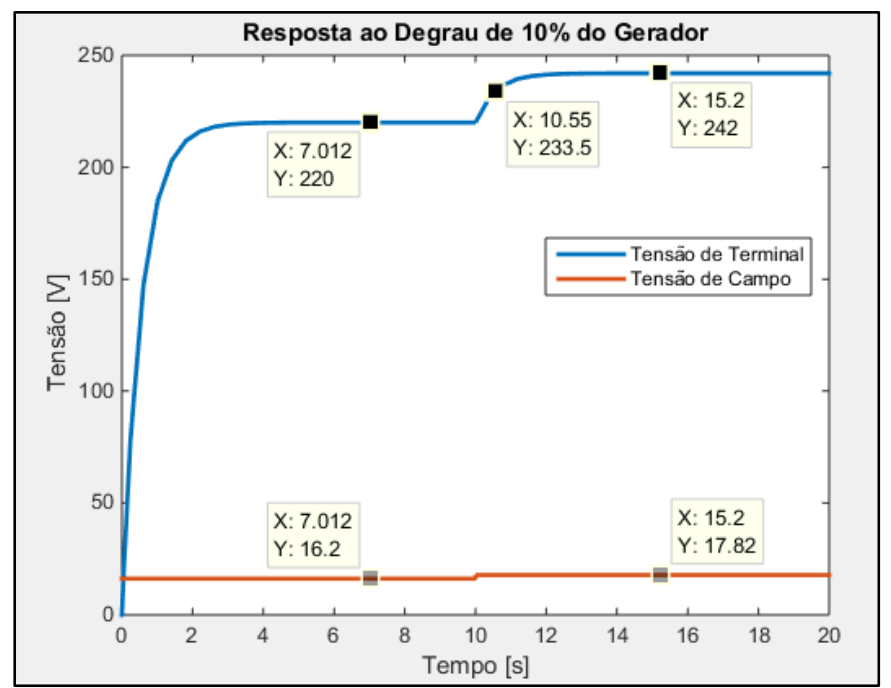

Figura 3. Representação do teste de resposta ao degrau

Substituindo os valores de $K_{g}$ e $T_{g}$ em (1), tem-se então o modelo matemático que representa a máquina síncrona utilizada neste trabalho, conforme apresenta (6).

$$
G(s)=\frac{13,58}{0,55 s+1}
$$

Como, nesse trabalho, o projeto de controle da excitação de campo do gerador depende dos valores de resistência e indutância da construção da bobina de campo da máquina, um teste de curva RL foi realizado para que obtessemos esses parâmetros.

Os dados referentes aos parâmetros do conversor CC-CC do tipo Buck, podem ser observados na Tabela 1. Substituindo os valores dos parâmetros da Tabela 1 em (3), obtém-se (7).

Tabela 1. Parâmetros físicos para a construção do modelo das dinâmicas

\begin{tabular}{ccc}
\hline Símbolo & Valor & Unidade \\
\hline$R$ & 30 & $\Omega$ \\
$L_{1}$ & 1,0 & $\mathrm{mH}$ \\
$L_{2}$ & 1,76 & $\mathrm{mH}$ \\
$C$ & 5600 & $\mu \mathrm{F}$ \\
$V_{\text {in }}$ & 32,30 & $V$ \\
$K_{g}$ & 13,58 & - \\
$\tau_{g}$ & 0,55 & $\mathrm{~S}$ \\
$f_{\text {chaveamento }}$ & $10 k$ & $\mathrm{~Hz}$ \\
$f_{\text {amostragem }}$ & $10 k$ & $\mathrm{~Hz}$ \\
\hline
\end{tabular}

$$
\frac{e_{f d}}{d}=\frac{5,768 \cdot 10^{6} s+9,832 \cdot 10^{10}}{s^{3}+1.705 \cdot 10^{4} s^{2}+2.8 \cdot 10^{5} s+3.044 \cdot 10^{9}}
$$

\subsection{Descrição do ambiente experimental}

\subsubsection{Grupo moto-gerador de 1,5 kVA}

O grupo Motor-Gerador utilizado, fabricado pela ENIKA LTDA., é formado por um motor CC, que simula uma fonte de energia primária e uma máquina síncrona de polos salientes, funcionando como gerador. A Figura 4 apresenta o motor CC acoplado à máquina síncrona por meio de um acoplamento inelástico.

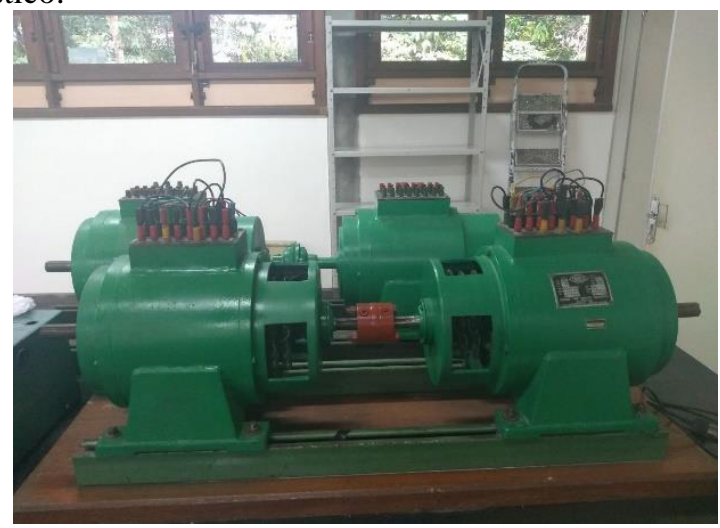

Figura 4. Grupo Moto-Gerador de 1,5 kVA localizado no LME (UFAM)

\subsubsection{Sistema de aquisição de tensão}

A medição do valor da tensão terminal do gerador síncrono é fundamental para a retroalimentação do controle da malha de tensão. Esta medição é feita através de um sistema de aquisição de tensão que fornece um valor de tensão proporcional ao valor eficaz da tensão terminal na saída do gerador. Este sistema realiza o condicionamento e filtragem do sinal medido. $\mathrm{O}$ esquema elétrico da malha de aquisição de tensão terminal é ilustrado na Figura 5.

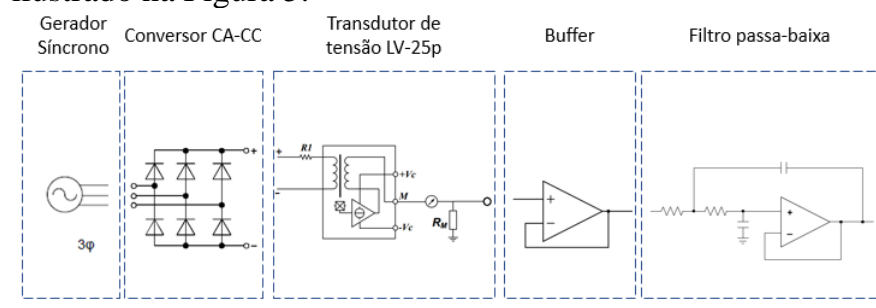

Figura 5. Malha do sistema de aquisição de tensão

\subsection{Projeto do controlador}

Para a realização do projeto do controlador, utilizou-se a técnica Lugar Geométrico das Raízes (LGR), de modo a obter os valores dos ganhos do controlador, de posse dos modelos matemáticos referentes a máquina síncrona e o sistema de excitação (conversor Buck), pode-se projetar um sistema de controle de estrutura proporcional integral (PI), conforme apresenta (8), que atenda as especificações de desempenho determinadas para o sistema de geração de energia elétrica, isto é, sobre-sinal máximo de $5 \%$ e tempo máximo de acomodação menor do que 2 segundos. 
Diferentes técnicas de controle foram propostas para analisar o sistema RAT com o objetivo de obter uma melhor resposta dinâmica. No entanto, entre esses controladores, o controlador PID é o controlador preferível devido à sua estrutura de projeto simples e robustez às variações dos parâmetros do sistema (EKINCI et al., 2019).

$$
C(s)=\frac{0.005 \cdot(s+2)}{s}
$$

Assim, os valores de ganho para o controlador, são de: $K p=0.005$ e $K_{i}=0.01$.

Dessa forma, utilizando o método de Tustin para a discretização do controlador, com freqüência de amostragem de $10 \mathrm{kHz}$, tem-se (9), a equação do controlador discretizado

$$
C d(z)=\frac{0.00505 z-0.00495}{z-1}
$$

Esse controlador é implementado na plataforma Arduino que fornece a informação de controle em um sinal PWM. A visão geral do sistema é apresentada na Figura 6.

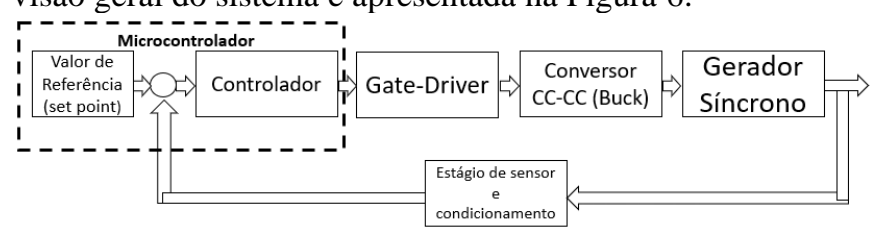

Figura 6. Visão geral do sistema

\subsection{Descrição dos testes realizados}

Com o intuito de avaliar o comportamento do sistema na presença do controlador, bem como validar o modelo matemático obtido para o sistema, desenvolveu-se um teste de variação da referência de tensão, objetivando avaliar o comportamento dinâmico do sistema na presença do controlador, comparando o modelo matemático obtido, com o sistema real desenvolvido, de modo a verificar se o controlador atendeu as especificações de desempenho especificados pelo projetista, o qual almeja-se: erro nulo de regime permanente e ter um sobressinal máximo inferior a $5 \%$ do valor final.

Deste modo, para realizar tal teste, realizou-se os seguintes passos: a referência inicia em zero e, primeiramente, é colocado como referência o valor de $220 \mathrm{~V}$; em seguida $240 \mathrm{~V}$; de novo em $220 \mathrm{~V}$; agora para $200 \mathrm{~V}$ e novamente $220 \mathrm{~V}$, cada mudança realizada com 10 segundos de diferença entre uma e outra.

\section{ANÁLISE DOS RESULTADOS}

Conforme apresentado na seção anterior, realizou-se os experimentos no sistema real e no sistema simulado, de modo a verificar as especificações de desempenho do sistema na presença do controlador.

Como o valor de referência para o gerador começa configurado em 0 (zero), então o circuito não inicia, ainda, a sua atuação sobre a máquina síncrona. Porém, os valores de tensão coletados no terminal do gerador, mostram uma tensão em torno de $13 \mathrm{~V}$ de valor eficaz, pois mesmo sem excitação na bobina, é característico do gerador essa resposta em velocidade nominal, como é mostrado na Figura 7.

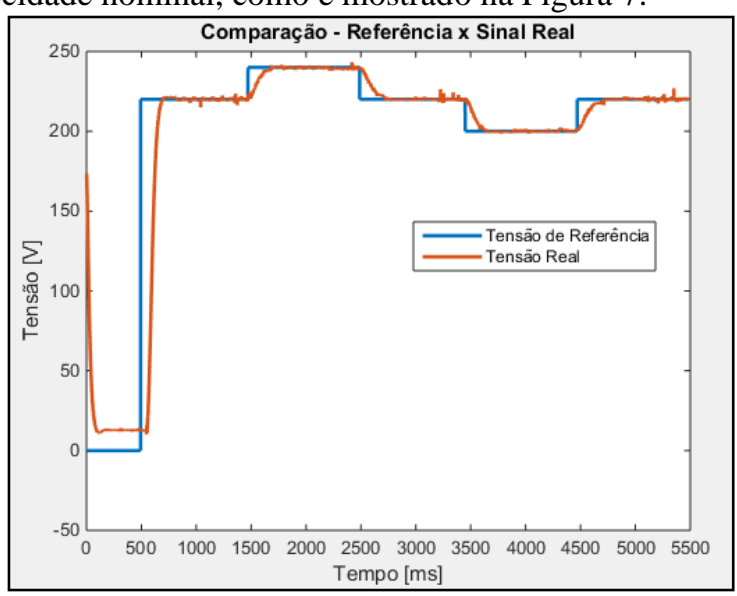

(9)

Figura 7. Comparação da tensão terminal com a tensão de referência experimental - RAT

Com o início da atuação do controle na excitação do gerador, devido à mudança no valor de referência desejado, a sua tensão de terminal sobe até atingir o valor determinado. Assim como no ambiente de simulação, a resposta é rápida e suave, apresentando sobre sinal máximo inferior a $2 \%$, bem como o tempo de assentamento de aproximadamente 2 segundos utilizando o critério de $2 \%$.

A Figura 8 apresenta o sinal de controle referente ao controle da excitatriz, que equivale ao ciclo de trabalho $(d)$ das chaves estáticas do conversor de potência CC-CC do tipo Buck.

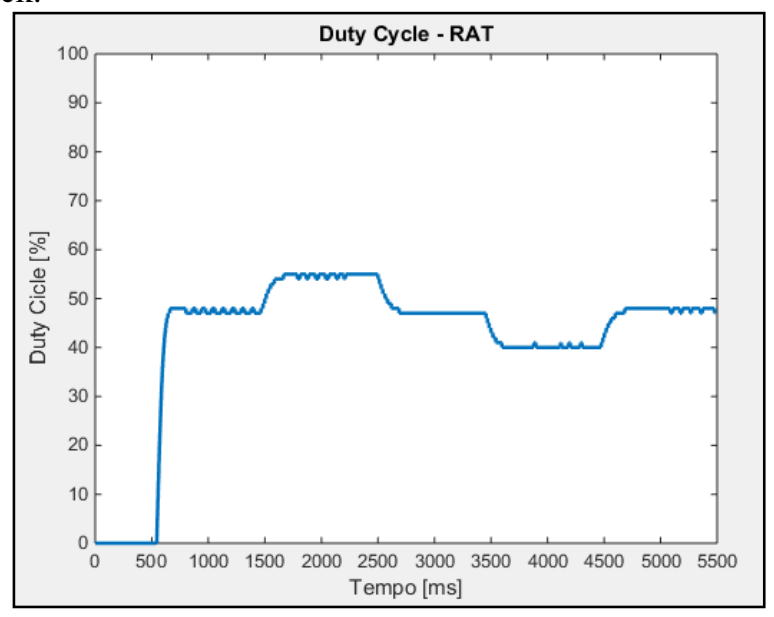

Figura 8. Ciclo de trabalho do sistema físico - RAT

Como é possível visualizar através dos gráficos de resposta obtidos, o regulador automático de tensão construído tem a sua funcionabilidade comprovada, visto que os valores de tensão na saída do gerador seguem os valores escolhidos como referência em cada situação.

Por fim, as respostas experimentais e de simulação são colocadas em um mesmo gráfico, e este é exibido na Figura 9. 


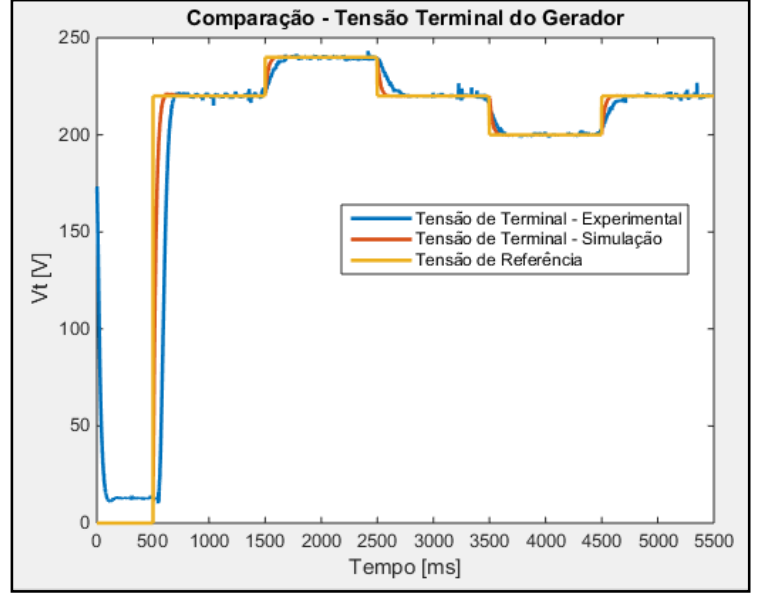

Figura 9. Comparação de Tensão Terminal

Esta visualização é particularmente importante para observar-se a diferença de velocidade entre um sistema construído em ambiente de simulação, onde são desconsiderados alguns efeitos na dinâmica dos modelos, com um ambiente desenvolvido experimentalmente. O ponto relevante de discordância entre os modelos, é o valor inicial em que está o nível de tensão do gerador quando a referência é zero. Pela construção do gerador, apenas a interação entre o rotor não-excitado girando em velocidade nominal e os enrolamentos de armadura, já produz um valor de tensão na saída; e essa dinâmica não é retratada no modelo de $1^{\mathrm{a}}$ ordem do gerador utilizado.

\section{CONCLUSÕES}

Foi apresentado, neste trabalho, a construção de um RAT utilizando a metodologia LGR para o projeto de controle utilizando uma estrutura PI, aplicado a uma máquina síncrona por meio de um sistema de excitação estático (conversor Buck) para uma máquina síncrona. A implementação física compreendeu a construção de um atuador, dedicado para excitação de campo, seguido de um sistema de condicionamento e filtragem.

Observando os resultados demonstrados dos ensaios, percebe-se o funcionamento adequado do controlador projetado, esperava-se um valor de overshoot de até $5 \%$ e o tempo de acomodação em regime permanente de até 2 segundos. O tipo de sistema trabalhado possui um comportamento inerentemente rápido, percebido nos testes em malha aberta, o que facilita a sua abordagem. Dessa forma, com resposta rápida, subida suave e valor de regime permanente adequado, seus parâmetros encontram-se dentro dos limites aceitáveis de implementação, como mostrado na seção de resultados.

\section{REFERÊNCIAS}

BARBI, I. (2015). Modelagem de Conversores CC-CC Empregando Modelo Médio em Espaço de Estados. Edição do Autor.
CALDERON, Jorge. (2017). Stability Enhancement of an Industrial Power System by AVR Gain Readjustment. IEEE Latin America Transactions, volume 15, 663-668

COSTA, A. C., AYRES JÚNIOR, F.A.C., NASCMENTO FILHO, P. S., MORAES, A. R. B. FARIA F. P., COSTA JÚNIOR, F. J, BARRA JÚNIOR, W. (2012). Sintonia de Controladores PID pelos Métodos de Ziegler- Nichols e Resposta em Frequência para a Regulação de Velocidade de um Sistema de Geração em Escala Reduzida de 10KVA. CBA, Campina Grande-PB, Brasil.

EKINCI, Serdar, HEKIMOGLU, Baran. (2019). Improved Kidney-Inspired Algorithm Approach for Tuning of PID Controller in AVR System. IEEE Access, volume 7, 39935 39947.

FERNANDES, Pablo Cravo. (2011). Regulador Automático de Tensão: Influência do Ganho no Sistema Elétrico de Potência. Dissertação de Mestrado. In; UNIFEI, Brasil.

GHAMRI, L. Y., AWADH, H., SHAMSI, N. A., ALKHATERI, S., KHURRAM, A., REHMAN, H. (2019). Robust AVR design for the synchronous generator. The Journal of Engineering, volume 2019, 4111-4115.

KUNDUR, P. (1994) Power System Stability and Control. McGraw-Hill.

MORAES, A. R. B. de. (2011). Desenvolvimento e Implementação de Estratégias de Controle Digital para Regulação de Tensão e Amortecimento de Oscilações Eletromecânicas em um Gerador Síncrono de $10 \mathrm{kva}$. Dissertação de Mestrado, In: UFPA, Brasil.

NASCIMENTO FILHO, P. S. (2011). Investigação de Estratégias de Controle Digital para Regulação de Velocidade e Emulação da Dinâmica de Turbinas Hidráulicas, com Implementação e Testes Experimentais em uma Micromáquina de 10 KVA. Dissertação de Mestrado,In: UFPA, Brasil

SAUER, P. W.; PAI, M. A. (1998). Power System Dynamics and Stability. Prentice Hall.

UMANS, Stephen D. (2014). Máquinas Elétricas de Fitzgerald e Kingsley. McGraw-Hill, $7^{\mathrm{a}} \mathrm{ed}$. 